\title{
Genetic markers for lactation persistency in primiparous Australian dairy cows
}

\author{
J. E. Pryce, ${ }^{* 1}$ M. Haile-Mariam, ${ }^{*}$ K. Verbyla, ${ }^{*} \dagger$ P. J. Bowman, ${ }^{*}$ M. E. Goddard, ${ }^{*} \dagger$ and B. J. Hayes \\ *Department of Primary Industries, 1 Park Drive, Bundoora, Victoria 3083, Australia \\ †Institute of Land and Food Resources, University of Melbourne, Parkville, Victoria 3010, Australia
}

\begin{abstract}
Good performance in extended lactations of dairy cattle may have a beneficial effect on food costs, health, and fertility. Because data for extended lactation performance is scarce, lactation persistency has been suggested as a suitable selection criterion. Persistency phenotypes were calculated in several ways: P1 was yield relative to an approximate peak, P2 was the slope after peak production, and P3 was a measure derived to be phenotypically uncorrelated to yield and calculated as a function of linear regressions on test-day deviations of days in milk. Phenotypes P1, P2, and P3 were calculated for sires as solutions estimated from a random regression model fitted to milk yield. Because total milk yield, calculated as the sum of daily sire solutions, was correlated to P1 and P2 $(r=0.30$ and 0.35 for $\mathrm{P} 1$ and $\mathrm{P} 2$, respectively), $\mathrm{P} 1$ and $\mathrm{P} 2$ were also adjusted for milk yield (P1adj and P2adj, respectively). To find genomic regions associated with the persistency phenotypes, we used a discovery population of 743 Holstein bulls proven before 2005 and 2 validation data sets of 357 Holstein bulls proven after 2005 and 294 Jersey sires. Two strategies were used to search for genomic regions associated with persistency: 1) persistency solutions were regressed on each single nucleotide polymorphism (SNP) in turn and 2) a genomic selection method (BayesA) was used where all SNP were fitted simultaneously. False discovery rates in the validation data were high $(>66 \%$ in Holsteins and $>77 \%$ in Jerseys). However, there were 2 genomic regions on chromosome 6 that validated in both breeds, including a cluster of $6 \mathrm{SNP}$ at around 13.5 to 23.7 Mbp and another cluster of 5 SNP (70.4 to $75.6 \mathrm{Mbp}$ ). A third cluster validated in both breeds on chromosome 26 (0.33 to $1.46 \mathrm{Mbp})$. Validating SNP effects across 2 breeds is unlikely to happen by chance even when false discovery rates within each breed are high. However, marker-assisted selection on these selected SNP may
\end{abstract}

Received August 23, 2009.

Accepted January 19, 2010.

${ }^{1}$ Corresponding author: jennie.pryce@dpi.vic.gov.au not be the best way to improve this trait because the average variation explained by validated SNP was only 1 to $2 \%$. Genomic selection could be a better alternative. Correlations between genomic breeding values predicted using all SNP simultaneously and estimated breeding values based on progeny test were twice as high as the equivalent correlations between estimated breeding values and parent average. Persistency is a good candidate for genomic selection because the trait is expressed late in lactation.

Key words: genomic selection, genome-wide association study, lactation persistency

\section{INTRODUCTION}

Persistency of lactation in dairy production can be defined in many different ways. In general, the rate of decline in production after peak milk production is considered to be a good indicator of persistency (Gengler, 1996). Furthermore, persistency is often adjusted for phenotypic level of milk yield because persistency in high yielding cows is not the same as persistency in low yielding dairy cows (Gengler, 1996). Thus, a flatter lactation curve without a decrease in total production is desirable (Jakobsen et al., 2002)

Although there has been speculation regarding the economic benefits of increased persistency (Cole and VanRaden, 2006), this trait could be used to select for extended lactations (Haile-Mariam and Goddard, 2008). Good performance in extended lactation may have a beneficial effect on food costs, health, and fertility (Dekkers et al., 1998). Swalve and Gengler (1999) suggested that persistency could lead to improvements in dairy cow welfare because cows with flatter lactation curves have lower energy deficits in early lactation and may experience a lower level of metabolic stress. However, these expectations were not confirmed by HaileMariam et al. (2003) in seasonal calving systems.

Gengler (1996) described several ways of defining persistency. Some methods are based on ratios of partial and total yields, and others are associated with the shape of the lactation curve (e.g., the rate of decline in milk yield from peak production). Novel approaches 
to calculating persistency include using eigenvectors derived from the genetic variance matrix obtained from random regression analysis to calculate a measure of persistency (e.g., Druet et al., 2005). Persistency EBV can, for example, be calculated as a byproduct of a testday model genetic evaluation (Jamrozik et al., 1998) or can be obtained by evaluating transformed solutions from similar methods as best prediction (e.g., Cole and VanRaden, 2006).

Persistency is an excellent target trait for genomic selection (Meuwissen et al., 2001) because it is sex limited, has a low to moderate heritability (Jakobsen et al., 2002; Cole and VanRaden, 2006; Haile-Mariam and Goddard, 2008; Cole and Null, 2009), and, for cows, phenotypes are available only after a full lactation has been completed.

High throughput genotyping of many SNP is now affordable in cattle, which has allowed genomic selection programs to be implemented in dairy cattle breeding programs (Harris et al., 2008; Hayes et al., 2009; VanRaden et al., 2009). For many quantitative traits, such as milk yield, fertility, and health, there are many loci that contribute to the genetic variance (e.g., Cole et al., 2009). Therefore, the genomic selection approach, which is based on markers distributed across the whole genome, is likely to be more successful than markerassisted selection, which is generally based on a small number of genetic markers (Hayes et al., 2009).

There were 2 main aims of this study. First, we identified genomic regions associated with variation in persistency and then validated these regions in 2 data sets consisting of 1) young Holstein sires, proven after 2005 and 2) Jersey bulls. Second, we assessed the accuracy of genomic breeding values (GEBV) for persistency using either all SNP fitted simultaneously or those SNP identified as being statistically significant in the single SNP regression method. The prediction equation was derived in the discovery data set and was then used to calculate breeding values in the validation data set (Holsteins only). The accuracies of the GEBV were then assessed based on the correlation between the GEBV and the EBV calculated using daughter phenotypic data.

\section{MATERIALS AND METHODS}

\section{Data}

Genetic markers were obtained for 294 Jersey and 1,100 Holstein sires using the BovineSNP50 BeadChip (Illumina, San Diego, CA; Matukumalli et al., 2009). A complete list of SNP names, locations, and SNP identifications (rs and ss numbers) can be found in Table S5 of Matukumalli et al. (2009). Single nucleotide polymorphisms were approximately equally spaced across the genome (at an average distance of $67 \mathrm{kbp}$ ). Single nucleotide polymorphisms were excluded from further analysis if they had a call rate of less than $90 \%$, had a minor allele frequency of less than $2.5 \%$, and violated Hardy-Weinberg expectations of genotype frequencies (Hardy-Weinberg chi-squared values of greater than 600). Single nucleotide polymorphisms that could not be mapped or that were on the $\mathrm{X}$ chromosome were excluded. The genotypes were then submitted to fastPhase (Scheet and Stephens, 2006) to impute missing genotypes for reconstruction of haplotypes from unphased SNP data. fastPhase is based on a cluster model for haplotypes and uses an expected-maximization algorithm to infer haplotypes, from which missing genotypes are imputed (Scheet and Stephens, 2006). The accuracy with which missing genotypes were imputed was $97.8 \%$ (Hayes et al., 2009). After editing, the number of SNP available for analysis was 39,048. The genotype editing criteria were chosen to exclude SNP with a high rate of genotyping error and those that were low frequency in the data set. Full details of the editing criteria, including genotype imputation, are given by Hayes et al. (2009)

First-parity records on milk volume were extracted from the Australian DHI Scheme database on cows that calved between 1999 and 2007. Haile-Mariam and Goddard (2008) reported that first-parity cows were more persistent than second-lactation cows, meaning that persistency should be analyzed within lactation. The data were edited to retain only herds with daughters of sires that had genotype information plus their contemporaries. Additionally, only herd-years with a minimum of 10 cows were kept. Also, to obtain estimates of lactation slopes for sires, only those with 30 or more daughters were included. The final data sets included first-lactation records of 797,025 cows sired by 3,459 sires for the Holstein-Friesian data set and 68,230 first-lactation records of daughters of 1,196 sires for the Jersey data set.

\section{Statistical Methods}

Sire solutions for third-degree Legendre polynomials fitted to first-lactation test-day records of milk yield were estimated using a random regression BLUP model in ASReml (Gilmour et al., 2006). A sire model was used in preference to an animal model because it allows adjusted daily yields to be estimated for each sire in the data. The (co)variance components and model used in the BLUP analysis were obtained from Haile-Mariam and Goddard (2008); their model was developed using a similar data set to the present study. The model used was 


$$
\begin{aligned}
y_{i j k l m}= & \mu+H T D_{i}+Y S_{j}+\sum_{n=1}^{3} A_{n} X_{k n}+\sum_{n=1}^{8} D_{n} Z_{k n} \\
& +\sum_{n=1}^{3} P_{m n} Z_{k n}+\sum_{n=1}^{3} S_{\ln } Z_{k n}+e_{i j k l m},
\end{aligned}
$$

where $y_{i j k l m}$ is the daily observation of milk yield (from $\mathrm{d}$ 5 to 305 of lactation); $\mu$ is the mean; $H T D_{i}$ is the effect of the $i$ th herd test-day; $Y S_{j}$ is the $j$ th year-season; $A_{n}$ is a fixed regression coefficient of the trait on the age at test; $X_{k n}$ is the observation of the $n$th order orthogonal polynomial corresponding to age on the $k$ th test-day; $D_{n}$ is a fixed regression coefficient of the trait on the DIM; $Z_{k n}$ is the observation of the $n$th order orthogonal polynomial corresponding to DIM on the $k$ th test-day; $P_{m n}$ is a random regression coefficient of the trait on DIM of the $m$ th cow; $\mathrm{S}_{l n}$ is a random regression coefficient of the trait on DIM of the $l$ th sire; and $e_{i j k l m}$ is the residual error term. No relationship matrix among sires was used.

Daily solutions for milk yield were calculated using the sire random regression solutions for sires that had been genotyped for SNP markers. These solutions were used as phenotypes in subsequent analyses. Three measures of persistency were calculated: $\mathbf{P} \mathbf{1}$, calculated as $\sum_{d=39}^{274} S_{i, d}-235 \times S_{i, 39}$ (similar to Harder et al., 2006, but assuming peak lactation was d 39 following Haile-Mariam and Goddard, 2008); P2, calculated as $S_{i, 54}-S_{i, 274}$ (similar to Jamrozik et al., 1998); and P3, calculated as $\sum^{300}$

$\sum_{d=5}^{300}\left(\mathbf{D}_{d}-\mathbf{D}_{0}\right)\left(\boldsymbol{\mu}_{\mathbf{d}}-\boldsymbol{\mu}_{\mathbf{0}}\right)$ (similar to the best prediction of persistency described by Cole and VanRaden, 2006). In all 3 measures of persistency, $S_{i}$ is sire solution of sire $i$ on day $d$ of lactation and $\mathbf{D}$ is a vector whose elements represent DIM. $\mathbf{D}_{0}$ is the balance point that is used so that the covariance between P3 and milk yield is uncorrelated; $\mathbf{D}_{0}$ was calculated as $\frac{\mathbf{D}^{\prime} \mathbf{V} \mathbf{1}}{\mathbf{1}^{\prime} \mathbf{V} \mathbf{1}}$, where $\mathbf{1}$ is a vector of 1 s and $\mathbf{V}$ accounts for the changing sire variance and covariance across lactation. The vector $\mu_{\mathrm{d}}$ contains daily milk solutions for each sire, whereas $\boldsymbol{\mu}_{0}$ is the sire solution corresponding to $\mathbf{D}_{\mathbf{0}}$. The measure P1 is yield relative to an approximate peak, whereas $\mathrm{P} 2$ is the gradient after peak lactation and P3 is a measure of persistency that is designed to be phenotypically uncorrelated to milk yield. In addition to the measures of persistency, $300 \mathrm{~d}$ milk yield solutions were calculated as the sum of daily yields estimated from the polynomial equations (from d 5 to 305 of lactation). Persistency measures adjusted for milk yield were also calcu- lated by regressing P1 and P2 on 300 d milk yield solutions ( $\mathbf{P} \mathbf{1}$ adj and $\mathbf{P} \mathbf{2} \mathbf{a d j}$, respectively).

The data sets for subsequent analyses were created by dividing the Holstein sire solution data set into 2 parts. The discovery data set included solutions for Holstein bulls that received their first proof before 2005 $(\mathrm{n}=743)$, and the first validation data set included Holsteins that were first proven after $2005(\mathrm{n}=357)$. A cut-off date of 2005 was chosen to ensure that there were enough sires in the discovery and validation data sets and also because equations formed in the genomic selection part of the analysis could be used for prediction of younger animals, because this type of forward prediction is most useful for practical selection purposes. The second validation data set comprised 294 Jersey sires of all ages.

In the SNP-by-SNP analyses, the traits were regressed on individual recoded SNP positions one at a time using ASReml (Gilmour et al., 2006) in the discovery data set and validation data sets separately. The sire solutions for persistency were modeled using

$$
y_{i j}=\mu+u_{i}+b g_{i}+e_{i j},
$$

where $y_{i j}$ is the solution for the $i$ th sire for the measures of persistency and $\mu$ is the mean. The variable $u_{i}$ is the breeding value component for the $i$ th sire, where $u \sim N\left(0, \mathbf{A} \sigma_{a}^{2}\right)$, A being the additive relationship matrix constructed from the pedigree and $\sigma_{a}^{2}$ being the additive genetic variance. The variable $b$ is the regression on SNP genotype; $g_{i}$ is 0,1 , or 2 if the sire is homozygous $(0,0)$, heterozygous $(0,1)$, or homozygous $(1,1)$ for the SNP; and $e_{i j}$ is the error term.

When testing the significance of association between an SNP and persistency, it is important to apply an appropriate threshold. For example, if a stringent probability is used as the threshold, then very few SNP will be significant. However, it is possible that some important mutations may be missed. If the threshold is very relaxed, then high false discovery rates are generally expected. We adopted a 2-stage approach where SNP were initially selected in the discovery population on the basis of their $P$-values. If the same SNP was significant in one of the validation populations, it was deemed to be validated. Very few SNP were selected when $P<$ 0.001 was chosen as the threshold to select SNP from the discovery data set. Therefore, the threshold chosen to select SNP from the discovery data set was $P<$ 0.005. A threshold of $P<0.05$ was chosen for the validation data set because only the SNP significant in the discovery data set were tested in the validation data set. The false discovery rate (FDR) was calculated as 
$R \times P / S$, where $R$ is the number of tests, $P$ is the $P$-value used to in the $F$-test, and $S$ is the number of SNP with significant $F$-statistics (i.e., the expectation of the number of false discoveries by chance divided by the actual SNP significant at this threshold). So $\mathrm{FDR}=(39,048 \times 0.005) / \mathrm{S}$ for S SNP discovered in the discovery population and $\mathrm{FDR}=(\mathrm{S} \times 0.05) / \mathrm{V}$ for $\mathrm{V}$ SNP significant in the validation population.

When SNP are validated in an independent population, there will be some SNP that are statistically significant in both discovery and validation populations by chance; this probability is described by the FDR. However, a limitation with using FDR to evaluate the success of genome-wide association studies is that only SNP that validate in exactly the same position as SNP in the discovery population are included in the calculation. Sometimes the LD between QTL and SNP varies between populations (e.g., a neighboring SNP in the validation population may be in higher $L D$ with the QTL than an SNP that was found to be significant in the discovery population).

The average variation $\left(\mathrm{R}^{2}\right)$ explained by the SNP that were statistically significant in the discovery population and tested in the Holstein and Jersey validation data sets was estimated using a function of the $F$-statistic $(F)$ for each of a total of $N$ SNP that were significant in the Holstein discovery population for $a$ animals, where

$$
\mathrm{R}^{2}=\left(\frac{\sum_{i=1}^{N} F}{N}-1\right) \times 100 \% \times \frac{1}{a} .
$$

The 39,048 SNP were also fitted simultaneously in a Bayes A model, similar to the model described by Meuwissen et al. (2001) and modified to include a polygenic effect (e.g., Hayes et al., 2009). The SNP effects were estimated in the discovery population and direct estimated genomic values (DGV) were calculated in the Holstein validation population, where the DGV of a sire is $\mathrm{DGV}=\sum_{\mathbf{j}=\mathbf{1}}^{\mathrm{m}} \mathbf{X} \hat{\mathbf{g}}, \mathbf{X}$ being an $m \times 1$ vector of genotypes ( 0 for homozygote 00,1 for heterozygote 01 , and 2 for homozygote 11) and $\hat{\mathbf{g}}$ being an $m \times 1$ vector of estimated SNP effects for the $m=39,048$ SNP from the mean of the posterior distributions for each SNP from BayesA. A second $\mathbf{X}$ matrix was constructed, where $m$ was formed using the SNP that had a significance level of $P<0.005$ in the SNP-by-SNP analysis of the Holstein discovery data set. This was to see wheth- er similar accuracies could be achieved through fitting a reduced number of selected SNP versus all of the SNP.

Sire EBV for persistency were required to correlate predictions based on marker information to predictions based on daughter or parent information (i.e., by using phenotypic data). The EBV were estimated by fitting the following model to the sire persistency solutions:

$$
y_{i j}=\mu+u_{i}+e_{i j} .
$$

The terms used in this model are described in model 2. Model 3 was also used to calculate the proportion of additive genetic variation relative to phenotypic variation of the sire persistency solutions. Because each sire solution for persistency included information from progeny, this ratio is larger than the heritability estimate.

A polygenic component from pedigree [parent-average EBV (i.e., half the sire plus dam EBV)] was added to the DGV to capture some of the genetic variance not captured by the SNP to form a GEBV. Because the reliabilities of PA and DGV differed, weights were applied using a function of the respective squared correlation with the EBV [e.g., $w_{1}=r(E B V, D G V)^{2} ; w_{2}$ $\left.=\mathrm{r}(\mathrm{EBV}, \mathrm{PA})^{2}\right]$ in the following way (based on Weigel et al., 1998):

$$
\begin{aligned}
& b_{1}=\frac{\left(1-\mathrm{wt}_{1}\right)}{1-\mathrm{wt}_{1} \mathrm{wt}_{2}}, \\
& b_{2}=\frac{\left(1-\mathrm{wt}_{2}\right)}{1-\mathrm{wt}_{1} \mathrm{wt}_{2}} .
\end{aligned}
$$

Then, GEBV $=b_{1} \mathrm{PA}+b_{2} \mathrm{DGV}$.

In a group of young bulls the EBV would be unknown, so this method demonstrates an ideal situation where appropriate weights are applied to the PA and DGV.

\section{RESULTS}

Using all Holstein sires, the mean (SE) proportion of total variance in sire solutions for P1, P2, P3, and milk volume explained by the genetic variance were 0.48 (0.08), 0.53 (0.07), 0.12 (0.45), and 0.50 (0.08), respectively. After adjusting for milk volume to form P1adj and P2adj, the proportion of total variation explained by genetic variation was $0.38(0.08)$ and 0.44 (0.08), respectively. Note that these estimates are not heritabilities because many daughters contribute to the sire solutions used to calculate the ratios. 
Table 1. Number of SNP found to be significant or in the same direction in Holstein discovery and Holstein and Jersey validation data sets and percentage of false discovery rates (FDR) for persistency traits (P1, P2, P3, P1adj, and P2adj) and milk volume (milk) regressed on individual SNP ${ }^{1}$

\begin{tabular}{lrrrrrr}
\hline Item & P1 & P2 & P3 & Milk & P1adj & P2adj \\
\hline Holstein discovery $P<0.005(\mathrm{n})$ & 340 & 302 & 309 & 738 & 357 & 262 \\
Holstein validation $P<0.05(\mathrm{n})$ & 16 & 22 & 18 & 152 & 18 & 20 \\
Holstein same direction (n) & 9 & 12 & 9 & 144 & 12 & 9 \\
Holstein same direction (\%) & 56 & 55 & 50 & 95 & 67 & 45 \\
Jersey $P<0.05(\mathrm{n})$ & 21 & 15 & 20 & 77 & 19 & 8 \\
Jersey same direction (n) & 13 & 7 & 7 & 37 & 10 & 3 \\
Jersey same direction (\%) & 62 & 47 & 35 & 48 & 53 & 38 \\
FDR discovery Holstein (\%) & 57 & 65 & 63 & 26 & 55 & 75 \\
FDR validation Holstein (\%) & 106 & 69 & 86 & 24 & 99 & 66 \\
FDR validation Jersey (\%) & 81 & 101 & 77 & 48 & 94 & 164 \\
\hline
\end{tabular}

${ }^{1}$ Persistency phenotypes were calculated in several ways: P1 was yield relative to an approximate peak, P2 was the slope after peak production, and P3 was a measure derived to be phenotypically uncorrelated to yield and calculated as a function of linear regressions on test-day deviations of days in milk (see text for calculations). Phenotypes P1, P2, and P3 were calculated for sires as solutions estimated from a random regression model fitted to milk yield. Because total milk yield, calculated as the sum of daily sire solutions, was correlated to P1 and P2, P1 and P2 were also adjusted for milk yield (P1adj and P2adj, respectively).

The number of significant SNP $(P<0.005)$ found for persistency in the discovery data set was 340,302 , and 309 for P1, P2, and P3, respectively (Table 1). This is equivalent to an FDR of 57,65 , and $63 \%$ for P1, P2, and P3, respectively. For milk volume, 738 SNP were significant at $P<0.005$ in the discovery data set, which

Table 2. Single nucleotide polymorphisms for P1adj that were $P<0.005$ in the Holstein discovery population and $P<0.05$ in Holsteins and Jerseys and, for Holsteins, were validated in the same direction in discovery and validation populations ${ }^{1}$

\begin{tabular}{|c|c|c|c|c|c|}
\hline Chromosome & Location bp & SNP name & $P$-value & $\begin{array}{l}\text { Direction } \\
\text { discovery }\end{array}$ & $\begin{array}{c}\text { Direction } \\
\text { validation }\end{array}$ \\
\hline \multicolumn{6}{|l|}{ Holstein } \\
\hline 6 & 74871316 & ARS-BFGL-NGS-69314 & 0.039 & + & + \\
\hline 6 & 75092837 & BTA-76827-no-rs & 0.006 & - & - \\
\hline 6 & 77941426 & Hapmap50522-BTA-98140 & 0.012 & - & - \\
\hline 7 & 107996289 & Hapmap40814-BTA-80565 & 0.010 & - & - \\
\hline 11 & 45805674 & ARS-BFGL-BAC-14274 & 0.032 & + & + \\
\hline 13 & 58782841 & ARS-BFGL-NGS-102408 & 0.021 & - & - \\
\hline 23 & 27162534 & ARS-BFGL-NGS-79310 & 0.020 & - & - \\
\hline 26 & 11162209 & UA-IFASA-8209 & 0.001 & - & - \\
\hline 26 & 11320618 & Hapmap52674-rs29020520 & 0.004 & - & - \\
\hline 26 & 14595382 & Hapmap52817-rs29011969 & 0.031 & + & + \\
\hline 27 & 6398901 & BTA-118887-no-rs & 0.023 & - & - \\
\hline 28 & 44915965 & BTA-122394-no-rs & 0.019 & + & + \\
\hline \multicolumn{6}{|l|}{ Jersey } \\
\hline 3 & 87783883 & BTA-68496-no-rs & 0.045 & - & + \\
\hline 6 & 72061135 & Hapmap24750-BTC-042016 & 0.013 & + & + \\
\hline 7 & 37274511 & ARS-BFGL-NGS-26917 & 0.035 & - & - \\
\hline 8 & 9540174 & BTA-44316-no-rs & 0.048 & + & - \\
\hline 9 & 55001487 & BTA-111931-no-rs & 0.044 & - & - \\
\hline 9 & 79835130 & ARS-BFGL-NGS-114465 & 0.015 & + & + \\
\hline 10 & 93336974 & ARS-BFGL-NGS-2949 & 0.003 & + & + \\
\hline 11 & 16998853 & BTB-00461926 & 0.033 & + & + \\
\hline 13 & 55329333 & ARS-BFGL-NGS-100973 & 0.044 & + & + \\
\hline 16 & 34940719 & BTA-38771-no-rs & 0.032 & - & - \\
\hline 16 & 71131076 & ARS-BFGL-NGS-99802 & 0.012 & + & - \\
\hline 17 & 2764114 & BTA-40708-no-rs & 0.021 & + & + \\
\hline 17 & 9096428 & ARS-BFGL-NGS-55629 & 0.037 & + & - \\
\hline 18 & 32630276 & ARS-BFGL-NGS-115751 & 0.028 & + & - \\
\hline 18 & 34866810 & ARS-BFGL-NGS-113568 & 0.023 & - & + \\
\hline 21 & 44075802 & Нарmap50902-ВTA-16342 & 0.001 & + & - \\
\hline 26 & 10810808 & ARS-BFGL-NGS-21794 & 0.032 & - & + \\
\hline 27 & 12383787 & ARS-BFGL-NGS-34295 & 0.007 & - & + \\
\hline 27 & 26593034 & BTA-62522-no-rs & 0.004 & - & - \\
\hline
\end{tabular}

${ }^{1}$ See text for definition of P1adj. 
Table 3. Single nucleotide polymorphisms for P2adj that were $P<0.005$ in the Holstein discovery population and $P<0.05$ in Holsteins and Jerseys and, for Holsteins, were validated in the same direction in discovery and validation populations ${ }^{1}$

\begin{tabular}{lrlccc}
\hline Chromosome & Location bp & SNP name & $P$-value & $\begin{array}{c}\text { Direction } \\
\text { discovery }\end{array}$ & $\begin{array}{c}\text { Direction } \\
\text { validation }\end{array}$ \\
\hline Holstein & & & & & \\
2 & 16000786 & BTB-01600593 & 0.017 & - & - \\
4 & 10139426 & ARS-BFGL-NGS-112143 & 0.038 & - & - \\
6 & 21593191 & ARS-BFGL-NGS-27962 & 0.017 & - & - \\
6 & 21620640 & UA-IFASA-1756 & 0.023 & - & - \\
8 & 11649044 & BTA-82896-no-rs & 0.041 & - & - \\
12 & 8844600 & Hapmap59058-rs29016195 & 0.026 & - & - \\
17 & 11271481 & BTA-42074-no-rs & 0.004 & - & - \\
18 & 64466895 & ARS-BFGL-NGS-38620 & 0.045 & + & + \\
26 & 3410026 & BTB-00920286 & 0.013 & + & + \\
Jersey & & & & & - \\
1 & 144105011 & ARS-BFGL-NGS-114541 & 0.042 & + & + \\
5 & 113682010 & ARS-BFGL-NGS-25555 & 0.043 & + & + \\
6 & 13520548 & BTA-12871-rs29024817 & 0.025 & + & + \\
6 & 21408490 & Hapmap45132-BTA-77765 & 0.003 & + & + \\
6 & 23744743 & Hapmap50782-BTA-78177 & 0.012 & + & + \\
13 & 70432390 & ARS-BFGL-NGS-35445 & 0.022 & + & - \\
\hline
\end{tabular}

${ }^{1}$ See text for definition of P2adj.

Table 4. Single nucleotide polymorphisms for P3 that were $P<0.005$ in the Holstein discovery population and $P<0.05$ in Holsteins and Jerseys and, for Holsteins, were validated in the same direction in discovery and validation populations ${ }^{1}$

\begin{tabular}{|c|c|c|c|c|c|}
\hline Chromosome & Location bp & SNP name & $P$-value & $\begin{array}{l}\text { Direction } \\
\text { discovery }\end{array}$ & $\begin{array}{c}\text { Direction } \\
\text { validation }\end{array}$ \\
\hline \multicolumn{6}{|l|}{ Holstein } \\
\hline 1 & 56015336 & ARS-BFGL-NGS-115305 & 0.045 & - & - \\
\hline 6 & 45423391 & ARS-BFGL-NGS-29047 & 0.007 & - & - \\
\hline 7 & 107996289 & Hapmap40814-BTA-80565 & 0.015 & - & - \\
\hline 7 & 108101557 & Hapmap57373-rs29019418 & 0.028 & - & - \\
\hline 11 & 45805674 & ARS-BFGL-BAC-14274 & 0.007 & + & + \\
\hline 11 & 63962517 & ARS-BFGL-NGS-47907 & 0.03 & + & + \\
\hline 17 & 11271481 & BTA-42074-no-rs & 0.013 & - & - \\
\hline 20 & 38539643 & ARS-BFGL-NGS-11884 & 0.041 & - & - \\
\hline 23 & 27162534 & ARS-BFGL-NGS-79310 & 0.011 & - & - \\
\hline 26 & 3298244 & BTB-00920216 & 0.048 & + & + \\
\hline \multicolumn{6}{|l|}{ Jersey } \\
\hline 1 & 156613986 & BTB-00072962 & 0.017 & + & + \\
\hline 2 & 138421341 & ARS-BFGL-NGS-60573 & 0.042 & - & + \\
\hline 2 & 139004087 & ARS-BFGL-NGS-17681 & 0.033 & + & - \\
\hline 2 & 139493014 & ARS-BFGL-NGS-111424 & 0.041 & - & - \\
\hline 5 & 15309838 & ARS-BFGL-NGS-22061 & 0.019 & + & - \\
\hline 5 & 15392995 & Hapmap39895-BTA-15668 & 0.012 & - & + \\
\hline 6 & 21408490 & Hapmap45132-BTA-77765 & 0.027 & + & + \\
\hline 9 & 101541463 & ARS-BFGL-NGS-102054 & 0.048 & - & - \\
\hline 14 & 2785212 & ARS-BFGL-NGS-103321 & 0.014 & - & - \\
\hline 17 & 66842129 & ARS-BFGL-NGS-23944 & 0.048 & + & - \\
\hline 18 & 19027348 & BTA-94677-no-rs & 0.009 & + & - \\
\hline 18 & 34866810 & ARS-BFGL-NGS-113568 & 0.013 & - & + \\
\hline 20 & 39697915 & Hapmap38412-BTA-50496 & 0.040 & - & + \\
\hline 20 & 39728148 & Hapmap53199-rs29014437 & 0.040 & + & - \\
\hline 20 & 40067078 & ARS-BFGL-NGS-5430 & 0.049 & + & - \\
\hline 20 & 42739807 & ARS-BFGL-BAC-33668 & 0.005 & - & + \\
\hline 24 & 55009334 & ARS-BFGL-NGS-13165 & 0.042 & + & + \\
\hline 26 & 3298244 & BTB-00920216 & 0.036 & + & - \\
\hline 26 & 9717437 & ARS-BFGL-NGS-19911 & 0.039 & + & - \\
\hline 29 & 42606575 & ARS-BFGL-NGS-11952 & 0.016 & - & - \\
\hline
\end{tabular}

${ }^{1}$ See text for definition of and calculation for P3. 
Table 5. Correlations of persistency EBV with parent average (PA), genomic breeding values (GEBV), and sire solutions (SLN $)^{1}$

\begin{tabular}{lccccc}
\hline Trait $^{2}$ & PA & GEBV1 & GEBV2 & GEBV3 & SLN \\
\hline P1 & 0.12 & 0.18 & 0.29 & 0.15 & 0.97 \\
P2 & 0.35 & 0.39 & 0.40 & 0.40 & 0.98 \\
P3 & 0.22 & 0.30 & 0.30 & 0.20 & 0.98 \\
Milk & 0.52 & 0.64 & 0.52 & 0.52 & 0.98 \\
P1adj & 0.15 & 0.27 & 0.21 & 0.29 & 0.96 \\
P2adj & 0.30 & 0.43 & 0.35 & 0.35 & 0.96 \\
\hline
\end{tabular}

${ }^{1}$ GEBV1 has all SNP effects plus the polygenic component fitted simultaneously; GEBV2 includes a reduced set of statistically significant $(P<0.005)$ SNP; GEBV3 was when the 5 largest SNP effects were fitted together.

${ }^{2}$ Persistency phenotypes were calculated in several ways: P1 was yield relative to an approximate peak, $\mathrm{P} 2$ was the slope after peak production, and P3 was a measure derived to be phenotypically uncorrelated to yield and calculated as a function of linear regressions on test-day deviations of days in milk (see text for calculations). Phenotypes P1, P2, and P3 were calculated for sires as solutions estimated from a random regression model fitted to milk yield. Because total milk yield, calculated as the sum of daily sire solutions, was correlated to P1 and P2, P1 and P2 were also adjusted for milk yield (P1adj and P2adj, respectively).

is an FDR of $26 \%$. After adjusting the persistency measures for milk yield, the FDR in the discovery data set were 55 and $75 \%$ for P1adj and P2adj, respectively.

In the validation data sets, the number of SNP that were significant $(P<0.005)$ in the discovery data set and significant $(P<0.05)$ in the validation data set ranged between $16(\mathrm{P} 1)$ and $22(\mathrm{P} 2)$ for Holsteins (Table 1). Between 8 (P2adj) and 21 (P1) SNP validated $(P<0.05)$ in Jerseys. The percentage of validated SNP with effects that were in the same direction as the corresponding SNP in the discovery population was between 45 and $67 \%$ for persistency traits in Holsteins. For milk volume, $95 \%$ of the SNP that validated in Holsteins were in the same direction as the discovery data set. For Jerseys, the percentage of validated SNP with effects in the same direction as the Holstein discovery data set was between 35 and $62 \%$ (i.e., not different from what would be expected by chance).

The SNP that were significant at $P<0.005$ in the Holstein discovery data set and validated in Holsteins or Jerseys at $P<0.05$ for P1adj, P2adj, and P3 are shown in Tables 2, 3, and 4. All validated SNP are shown for Jerseys, whereas only those SNP that validated in the same direction as the discovery population are shown for Holsteins because changes in phase between marker and QTL are unlikely to happen within breed. There were few SNP that validated in both Holsteins and Jerseys. A SNP on chromosome 26 (located at 3,298,224 bp) validated in both Holsteins and Jerseys for P3. This SNP was colocated with a cluster of SNP that validated in both Holsteins and Jerseys for P1adj, P2adj, and P3 across a region from 0.33 to $1.46 \mathrm{Mbp}$. There were also
2 SNP clusters on chromosome 6, including a SNP that validated in Jerseys on chromosome 6 at 23,744,713 bp in both P3 and P2adj. This SNP was colocated with a cluster of SNP that validated in both Holsteins and Jerseys for P2adj and P3 across a region of 21.4 to 23.7 Mbp (Figure 1). The other cluster on chromosome 6 was located between 70.4 and $77.9 \mathrm{Mbp}$ and was present only in P1adj and P2adj (Figure 1).

The average amount of variation explained by the SNP that were significant in the discovery data set (after adjusting for the variation explained by all SNP) was between 0.93 and $1.0 \%$ per SNP for persistency traits in Holsteins and 1.4 and $1.7 \%$ in Jerseys.

The 5 largest SNP were selected on the basis of the probability (from individual $F$-tests) of the regression of persistency on each SNP in turn in the discovery population. These SNP were fitted simultaneously in a multiple regression model in ASReml (Gilmour et al., 2006) to estimate SNP effects. Genomic breeding value 3 was calculated by combining the parent average with the respective DGV estimated by summing the 5 largest solutions from the multiple regression analysis multiplied by genotype $(0,1$, or 2$)$ for each bull. The correlations between the GEBV3 and EBV estimated using 5 SNP solutions were 0.15, 0.40, 0.20, 0.29, and 0.35 for P1, P2, P3, P1adj, and P2adj, respectively. With the exception of P3, these correlations exceeded the corresponding correlation between EBV and parent averages (Table 5).

For the BayesA analysis, SNP effects were estimated using data on all the sires in the Holstein discovery data set and GEBV calculated on a subset of 74 Holstein bulls from the validation data set. The subset from the validation data set included sires with more than 75 daughters/sire (e.g., reliable sire proofs).

The correlation between persistency EBV and GEBV ranged from 0.18 to 0.43 when all SNP were fitted (GEBV1, Table 5). When SNP were selected on the basis of the $P$-value of the regression of the phenotype on each SNP in turn $(P<0.005)$ in the Holstein discovery data set, the correlations between GEBV and EBV were between 0.21 and 0.40 (GEBV2). In all cases, the correlation between the GEBV and EBV exceeded the correlation between the EBV and PA.

To compare the locations of the SNP with significant associations from the genome-wide association study and the SNP with the largest effect in the BayesA analysis, the same number of BayesA SNP were selected as validated in both Holsteins and Jerseys (i.e., the number of SNP in Tables 3 and 4). The positions of the BayesA SNP with the largest effects for persistency are shown in Figures 2, 3, and 4 in addition to the size and positions of the single SNP analysis. The BayesA solutions appear to be more evenly spread across the 


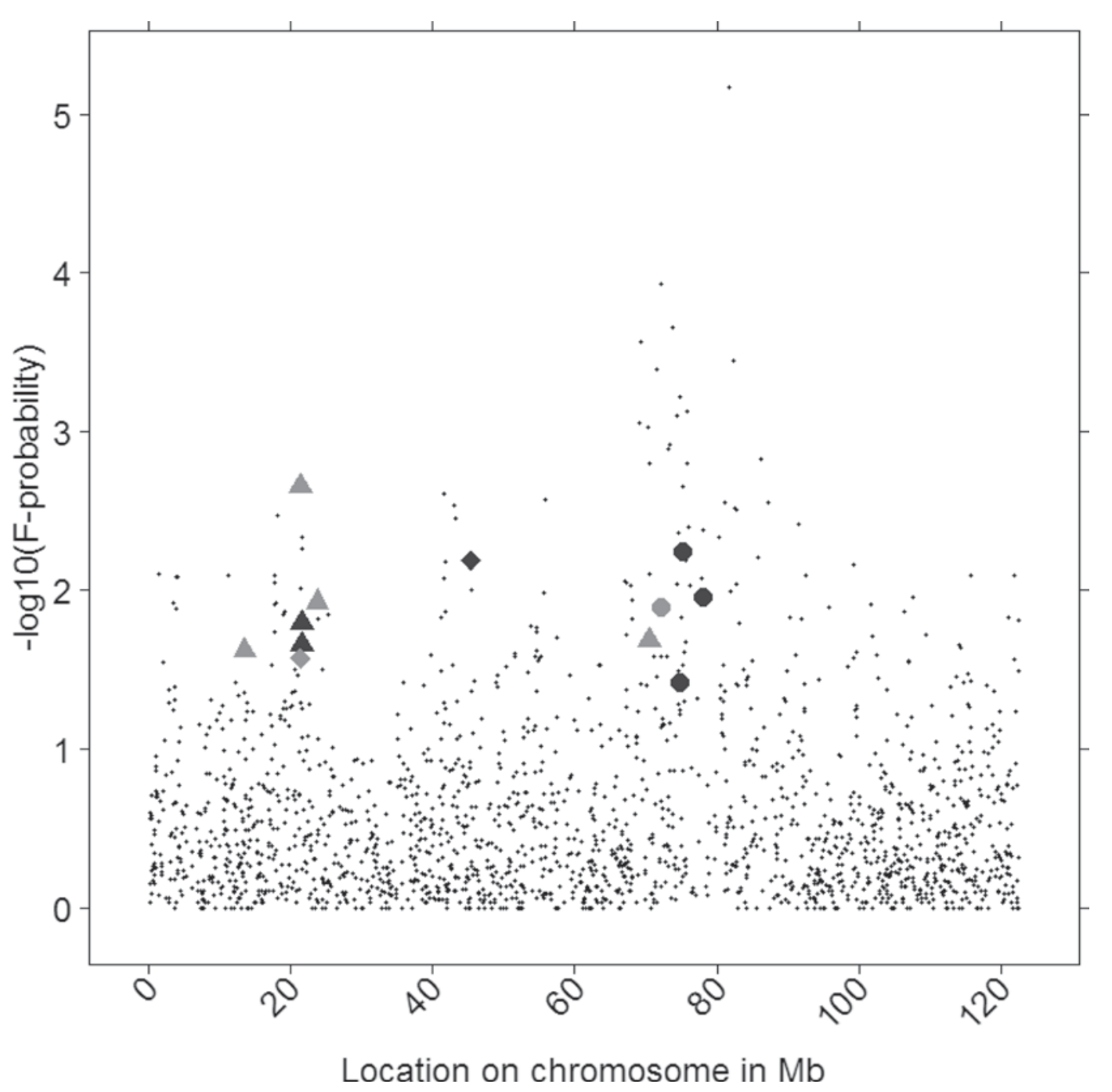

Figure 1. $-\log 10$ (F-probability) of SNP effects on chromosome 6 for P1adj (•), P2adj (ム), and P3 (•). Black shapes are SNP validated in Holsteins; gray shapes are SNP validated in Jerseys; small dots are all SNP effects in discovery Holsteins. See text for definition of P1adj, P2adj, and P3 and for the calculation for P3. Color version available in online PDF.

genome than the single SNP effects. However, some of the Bayes effects colocate with the validated SNP from the single SNP analysis (Figures 2, 3, 4, and 5), providing further evidence that the existence of a QTL in that part of the genome was real. For example, for P1adj (Figure 2), clusters of large BayesA SNP effects aligned with significant single SNP regression estimates on chromosomes 6 and 26; there are also single SNP effects that validated in Jerseys close to the plotted BayesA estimates on chromosomes 3 and 16. Again, the existence of large BayesA estimates at these positions gives more credibility to the existence of mutations affecting lactation persistency at these positions, although the evidence is not as convincing as the clusters on chromosomes 6 and 26. The positions and sizes of SNP validated for milk volume are shown in Figure 5; the largest SNP effects were found on chromosomes 6 , 14 , and 20.

\section{DISCUSSION}

In this study we describe panels of SNP validated in 2 breeds of dairy cattle that could be used to select for improved persistency of lactation. On chromosomes 6 and 26, there were SNP in close physical proximity that validated in Holsteins and Jerseys for P1adj, P2adj, and P3 (Figures 2, 3, and 4). Futhermore, there was reasonably good alignment between these clusters and the largest BayesA effects. This suggests that the power of our experiment was sufficient to detect genomic regions of importance for persistency. Furthermore, validating SNP across 2 breeds appears to be a powerful way of 


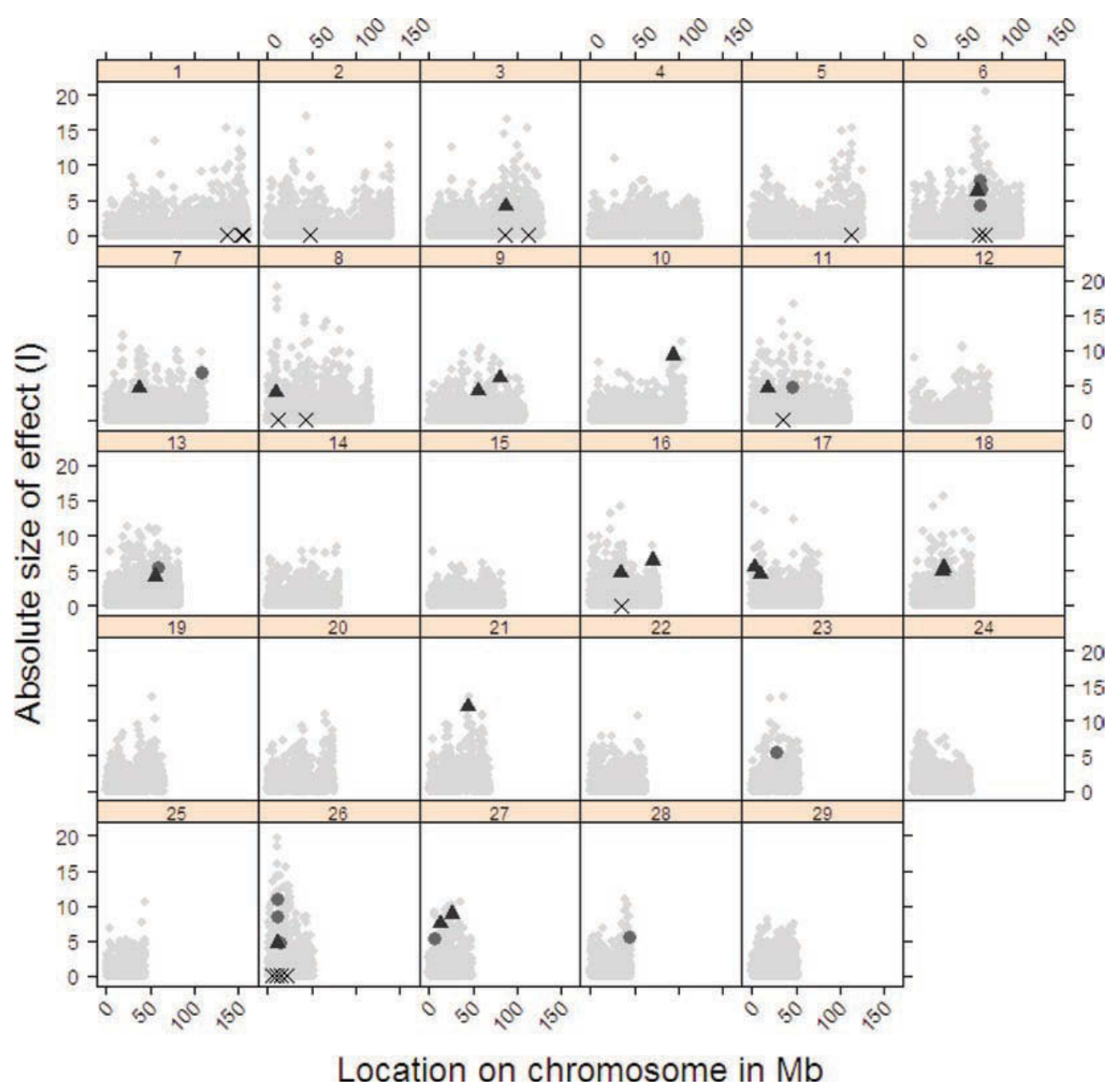

Figure 2. Solutions for P1adj by chromosome, size of effects in Holstein discovery data set (small gray dots), validated SNP in Holsteins (large gray dots), validated SNP in Jerseys (gray triangles), and positions of largest Bayes estimate (black crosses). See text for definition of P1adj. Color version available in online PDF.

distinguishing between false discoveries and SNP that genuinely have an effect on a trait of interest.

However, the density of SNP was not sufficient to find individual marker associations that persisted across breed. For example, the direction of marker effects was the same in both breeds for 1 SNP only. Jerseys have diverged from Holsteins over a large number of generations and both populations have small effective population sizes, so the linkage disequilibrium (LD) phase between the SNP and the QTL affecting persistency could be different in the 2 populations even at short distances. de Roos et al. (2008) investigated the extent of LD phase between Jerseys and Holsteins and concluded that LD phase would be conserved only between markers and QTL if the distance between them was less than $10 \mathrm{kbp}$. This is a much shorter distance than the average marker spacing in this study (67 kbp).
Therefore, it is likely that recombination events will have occurred between markers and the QTL we have detected since the divergence of the Holstein discovery and Jersey validation population, thus changing the phase between SNP and QTL. However, in Holsteins the phase could be expected to be conserved across the discovery and validation populations because the time of divergence is very short. For milk yield, for example, the percentage of SNP with the same direction in the Holstein discovery and Holstein validation population was $95 \%$. For the persistency traits, the figure was lower, likely a reflection of the higher FDR for these traits, in turn reflecting the lower power to detect associations for lower heritability traits.

Results from the BayesA analysis suggest this is a useful approach to QTL mapping. The largest BayesA effects lined up reasonably well with the single SNP 


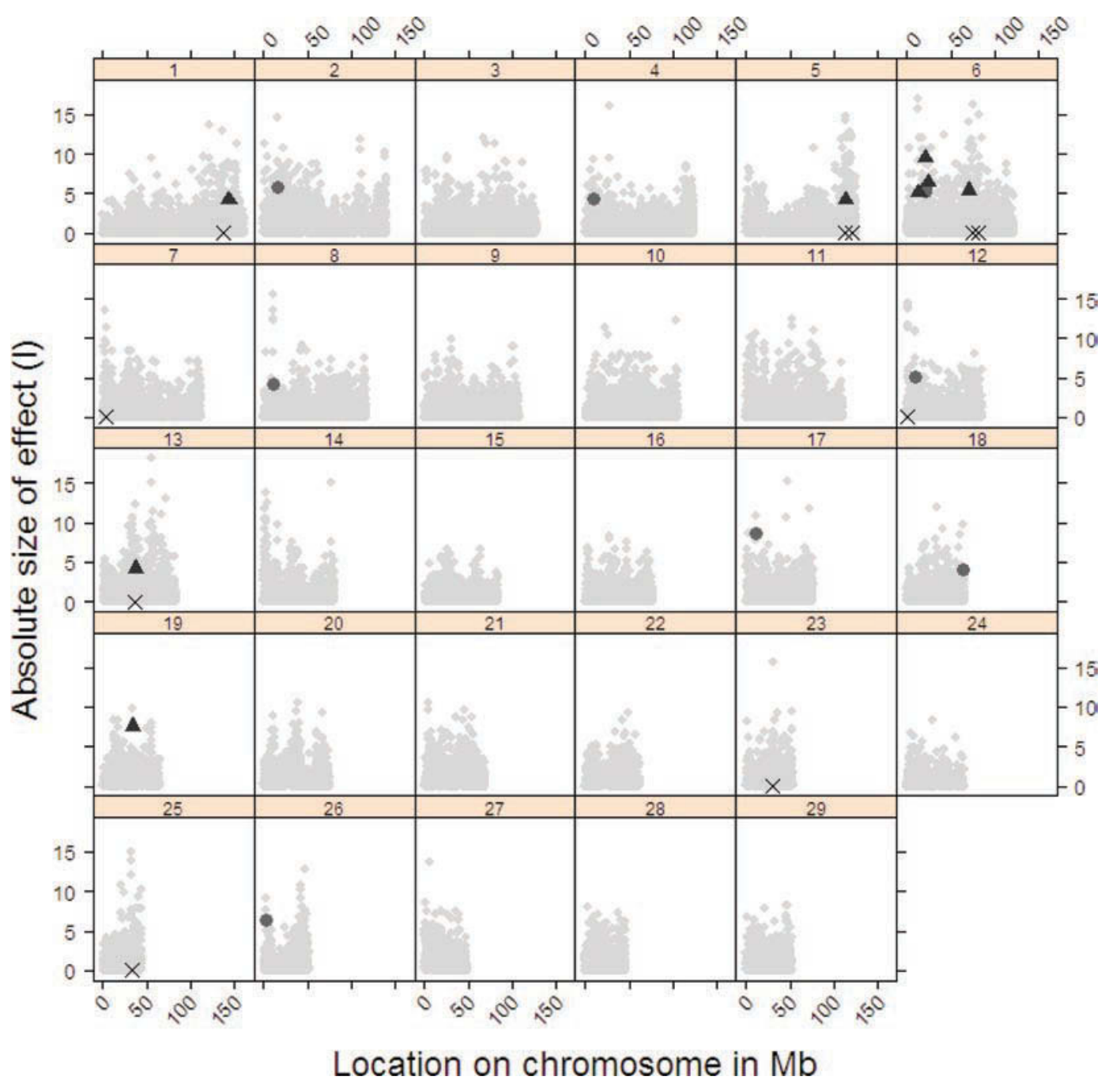

Figure 3. Solutions for P2adj by chromosome, size of effects in Holstein discovery data set (small gray dots), validated SNP in Holsteins (large gray dots), validated SNP in Jerseys (gray triangles), and positions of largest Bayes estimate (black crosses). See text for definition of P2adj. Color version available in online PDF.

solutions (Figures 2, 3, 4, and 5). Furthermore, even though there were SNP in opposing direction in the validated set of SNP that were significant for both Holstein populations (Table 1), the ones in close proximity to the large BayesA effects were always in the same direction. The BayesA method fitted all SNP simultaneously, whereas each SNP was fitted in turn in the SNP by SNP analysis. The SNP by SNP analysis will therefore potentially identify clusters of SNP in high LD with each as well as the QTL. The BayesA analysis, on the other hand, will lead to the selection of SNP with the highest level of association with the QTL, and once this effect is fitted the effect of surrounding SNP in high LD will be zero. This should result in smaller confidence intervals for the QTL location (e.g., ter Braak et al., 2005).
When we used the 5 most significant SNP to calculate GEBV in Holsteins, the correlations with EBV following progeny testing exceeded parent average alone for all traits except P3. These correlations are an indication of the accuracy of the GEBV, and therefore of how successful marker-assisted selection might be in improving persistency. However, for most traits, GEBV predicted using all (genome-wide) SNP (GEBV1) were more highly correlated with the EBV following progeny testing (Table 5), suggesting the genomic selection approach would result in higher genetic gains. It should be noted that because these correlations use the GEBV and EBV of only 74 sires, their associated errors are likely to be high.

The correlations between the GEBV using all SNP and persistency EBV in the Holstein validation popu- 


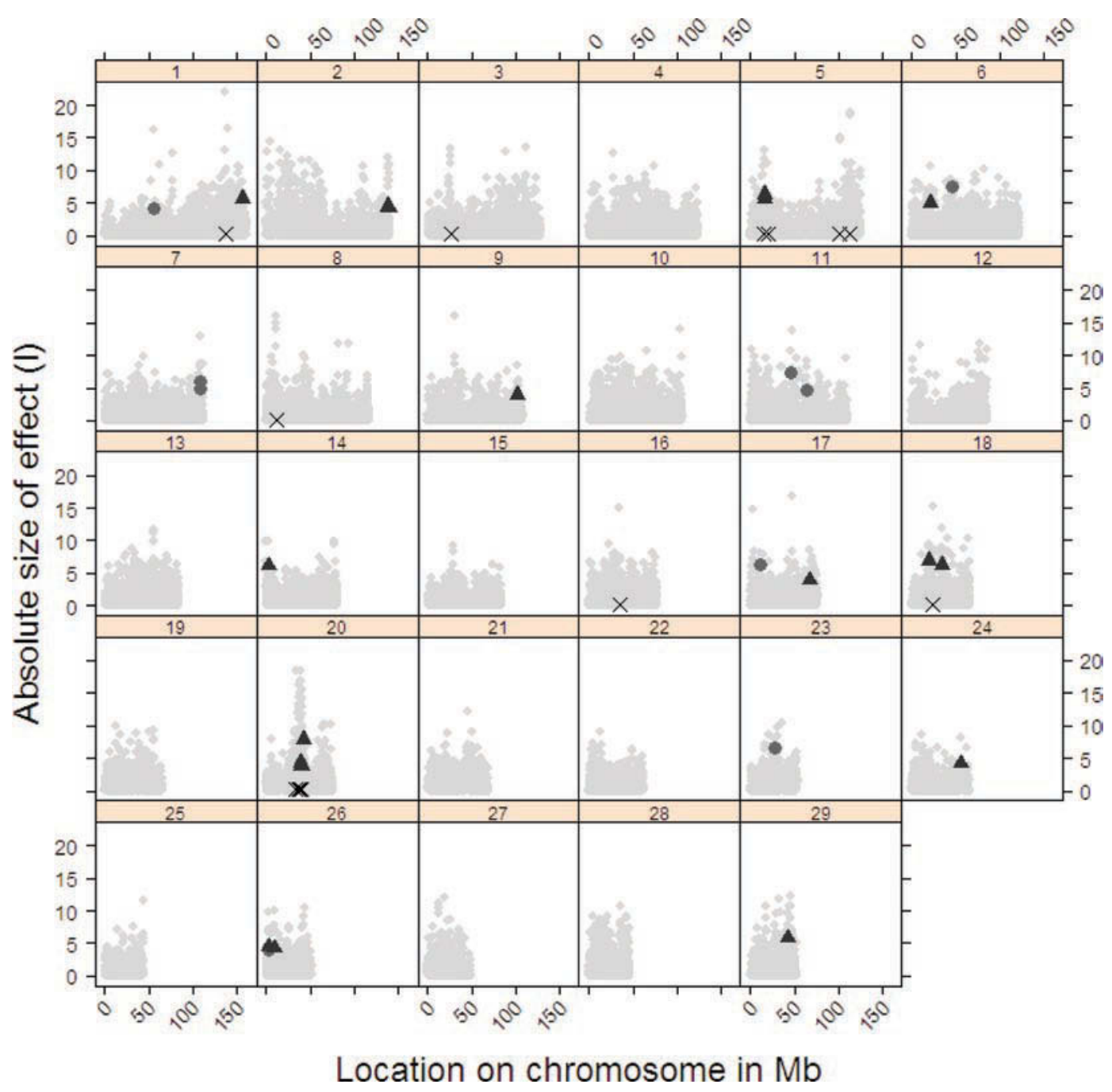

Figure 4. Solutions for P3 by chromosome, size of effects in Holstein discovery data set (small gray dots), validated SNP in Holsteins (large gray dots), validated SNP in Jerseys (gray triangles), and positions of largest Bayes estimate (black crosses). See text for definition and calculation of P3. Color version available in online PDF.

lation were between 0.18 and 0.43 (Table 5). These correlations are lower than for protein, fat, and milk volume (Hayes et al., 2009), probably reflecting the low to moderate heritability of persistency (Jakobsen et al., 2002; Cole and VanRaden, 2006; Haile-Mariam and Goddard, 2008; Cole and Null, 2009). Furthermore, the correlations between GEBV2 and EBV estimated using SNP selected from the single SNP analysis $(P<0.005$ in the Holstein discovery) [i.e., between 262 and 357 SNP (Table 1)] were very close to or exceeded the correlations estimated using all SNP (Table 5), provided that the effect of the SNP in the subset were estimated simultaneously with BayesA. This suggests that an adequate prediction of persistency can be obtained using around 300 SNP. Usai et al. (2009) reported that for production traits the accuracy of GEBV does not increase substantially when the number of SNP exceeds 1,000. For P1, P2, and P2adj, the correlation between the GEBV and EBV were actually higher using the reduced set of SNP than when all the SNP were fitted simultaneously. This may reflect the relatively low number of phenotypic records used in our study; if a larger number of records were available smaller effects could be estimated more precisely, so the number of SNP used to predict persistency GEBV could increase.

If the causative mutations for the SNP associations for persistency could be found, it would give important insights into the biology underlying this trait. The cluster of SNP discovered and validated in both breeds for P2adj and P3 on chromosome 6 was located at approximately 21.4 to $23.7 \mathrm{Mbp}$. This is a distance of approximately $50 \mathrm{Mbp}$ from the cluster of SNP that 


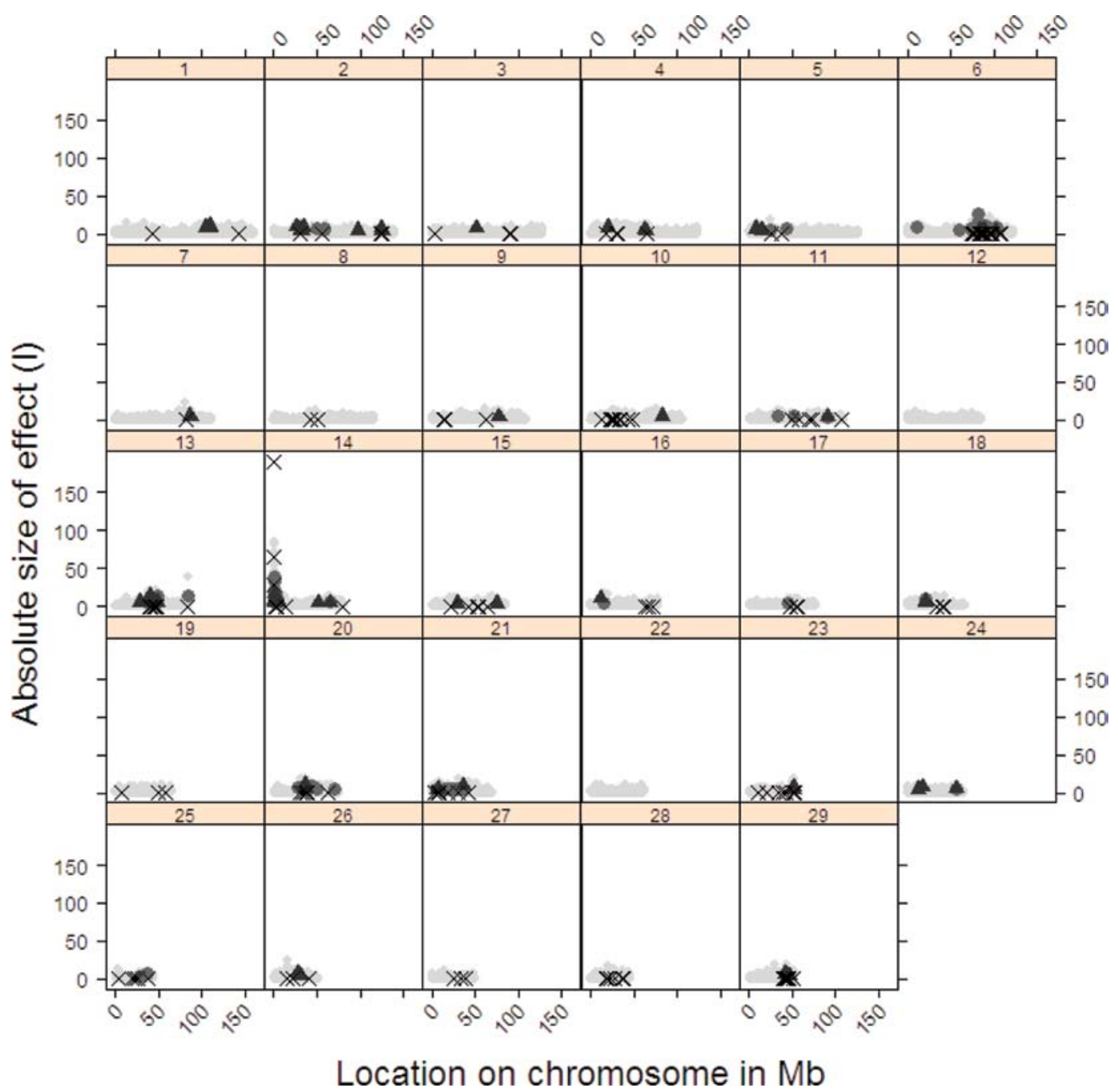

Figure 5. Solutions for milk volume by chromosome, size of effects in Holstein discovery data set (small gray dots), validated SNP in Holsteins (large gray dots), validated SNP in Jerseys (gray triangles), and positions of largest Bayes estimate (black crosses). Color version available in online PDF.

validated for milk yield on chromosome 6 , so the underlying mutations are unlikely to be the same. Candidate genes in this region (from BTA4.0; www.ensembl.org) include pyrophosphatase 2 , tachykinin receptor 3 , and mannosidase, $\beta$ A. Both pyrophosphatase 2 and mannosidase are expressed in the mammary gland, and tachykinins have a role in modulating prolactin release during lactation (Pisera et al., 1998).

The other cluster of SNP associated with P1adj and P3 was located at 70.4 to $77.9 \mathrm{Mbp}$ of chromosome 6. Other regions, such as SNP at the proximal end of chromosome 26 in P1adj, P2adj, and P3 (Figures 2, 3 , and 4), are also promising candidates for markers affecting persistency. However, there were no obvious candidate genes in these regions.

\section{CONCLUSIONS}

Several genomic regions containing SNP that are promising markers for lactation persistency in dairy cows have been identified. These include 2 genomic regions on chromosome 6 and 1 region of chromosome 26 . Using 2 validation populations, one being the same breed as the discovery data set and the other an alternative breed, was a powerful way to validate SNP discovered using genome-wide association studies and to minimize the risk of pursuing incorrect genomic regions when FDR in either of the individual breeds were high. Correlations between GEBV predicted using all SNP simultaneously and EBV based on progeny test were twice as high as the equivalent correlations 
between EBV and parent average. Persistency is a good candidate for genomic selection because the trait is expressed late in lactation.

\section{ACKNOWLEDGMENTS}

The authors thank the Geoffrey Gardiner Dairy Foundation Ltd. (Melbourne, Australia) for funding this research under grant BMP-08-003 "Improving feed conversion efficiency and lifetime profitability of the Australian dairy herd through genetic markers and biomarkers." We also acknowledge the helpful comments provided by 2 anonymous reviewers.

\section{REFERENCES}

Cole, J. B., and D. J. Null. 2009. Genetic evaluation of lactation persistency for five breeds of dairy cattle. J. Dairy Sci. 92:2248 2258.

Cole, J. B., and P. M. VanRaden. 2006. Genetic evaluation and best prediction of lactation persistency. J. Dairy Sci. 89:2722-2728.

Cole, J. B., P. M. VanRaden, J. R. O'Connell, C. P. Van Tassell, T. S. Sonstegard, R. D. Schnabel, J. F. Taylor, and G. R. Wiggans. 2009. Distribution and location of genetic effects for dairy traits. J. Dairy Sci. 92:2931-2946.

de Roos, A. P. W., B. J. Hayes, R. Spelman, and M. E. Goddard 2008. Linkage disequilibrium and persistence of phase in Holstein Friesian, Jersey and Angus cattle. Genetics 179:1503-1512.

Dekkers, J. C. M., J. H. Ten Hag, and A. Weersink. 1998. Economic aspects of persistency in dairy cattle. Livest. Prod. Sci. 53:237252

Druet, T., F. Jaffrezic, and V. Ducrocq. 2005. Estimation of genetic parameters for test day records of dairy traits for the first three lactations. Genet. Sel. Evol. 37:257-271.

Gengler, N. 1996. Persistency of lactation yields. A review. Interbull Bull. 12:87-96.

Gilmour, A. R., B. J. Gogel, B. R. Cullis, S. J. Welham, and R. Thompson. 2006. ASReml User Guide. Release 2.0. VSN International Ltd., Hemel Hempstead, UK.

Haile-Mariam, M., P. J. Bowman, and M. E. Goddard. 2003. Genetic and environmental relationship among calving interval, survival, persistency of milk yield and somatic cell count in dairy cattle. Livest. Prod. Sci. 80:189-200.

Haile-Mariam, M., and M. E. Goddard. 2008. Genetic and phenotypic parameters of lactations longer than 305 days (extended lactations). Anim. 2:325-335.

Harder, B., J. Bennewitz, N. Reinsch, G. Thaller, H. Thomsen, C. Kuhn, G. Erhardt, M. Forster, F. Reinhardt, and E. Kalm. 2006.
Mapping of quantitative trait loci for lactation persistency traits in German Holstein dairy cattle. J. Anim. Breed. Genet. 123:8996.

Harris, B. L., D. L. Johnson, and R. J. Spelman. 2008. Genomic selection in New Zealand and the implications for national genetic evaluation. Pages 325-330 in Proc. 36th ICAR Biennial Session, Niagara Falls, NY. Interbull Bull., Uppsala, Sweden.

Hayes, B. J., P. J. Bowman, A. J. Chamberlain, and M. E. Goddard. 2009. Genomic selection in dairy cattle: Progress and challenges. J. Dairy Sci. 92:433-443.

Jakobsen, J. H., P. Madsen, J. Jensen, J. Pedersen, L. G. Christensen, and D. A. Sorensen. 2002. Genetic parameters for milk production and persistency for Danish Holsteins estimated in random regression models using REML. J. Dairy Sci. 85:1607-1616.

Jamrozik, G., J. Jansen, L. R. Schaeffer, and Z. Liu. 1998. Analysis of persistency of lactation calculated from a random regression test day model. Interbull Bull. 17:64-69. http://www-interbull.slu.se/ bulletins/bulletin17/Jamrozik.pdf Accessed June 30, 2009.

Matukumalli, L. K., C. T. Lawley, R. D. Schnabel, J. F. Taylor, M. F. Allan, M. P. Heaton, J. O'Connell, S. S. Moore, T. P. L. Smith T. S. Sonstegard, and C. P. Van Tassell. 2009. Development and characterization of a high density SNP genotyping assay for cattle. PLoS ONE 4:e5350.

Meuwissen, T. H. E., B. J. Hayes, and M. E. Goddard. 2001. Prediction of total genetic value using genome-wide dense marker maps. Genetics 157:1819-1829.

Pisera, D. S. Theas, A. De Laurentiis, M. Lasaga, B. Duvilanski, and A. Seilicovich. 1998. The hormonal status modulates the effect of neurokinin A on prolactin secretion in female rats. J. Endocrinol. 159:389-395.

Scheet, P., and M. A. Stephens. 2006. A fast and flexible statistical model for large-scale population genotype data: Applications to inferring missing genotypes and haplotypic phase. Am. J. Hum. Genet. 78:629-644.

Swalve, H. H. and N. Gengler, 1999. Genetics of lactation persistency. Pages 75-82 in Metabolic Stress in Dairy Cows. J. D. Oldham, G. Simm, A. F. Groen, B. L. Nielsen, J. E. Pryce, and T. L. J. Lawrence, ed. BSAS occasional publication 24. Br. Soc. Anim. Sci. Penicuik, UK

ter Braak, C. J., M. P. Boer, and M. C. Bink. 2005. Extending Xu's Bayesian model for estimating polygenic effects using markers of the entire genome. Genetics 170:1435-1438.

Usai, M. G., B. J. Hayes, and M. E. Goddard. 2009. LASSO with cross-validation for genomic selection. Genet. Res. 91:427-436.

VanRaden, P. M., C. P. Van Tassell, G. R. Wiggans, T. S. Sonstegard, R. D. Schnabel, J. F. Taylor, and F. S. Schenkel. 2009. Invited review: Reliability of genomic predictions for North American Holstein bulls. J. Dairy Sci. 92:16-24.

Weigel, K. A., T. J. Lawlor Jr., P. M. VanRaden, and G. R. Wiggans, 1998. Use of linear type and production data to supplement early predicted transmitting abilities for productive life. J. Dairy Sci. 81:2040-2044 